\title{
Extension of the Euler-Savary Equation to Hypoid Gears*
}

\author{
Norio ITO** and Koichi TAKAHASHI ${ }^{* * *}$
}

\begin{abstract}
The Euler-Savary equation is the most fundamental relationship used to determine the relative curvature of a two-dimensional tooth profile which corresponds to relative motion. In this paper, the necessary conditions for gearing were analyzed, when a pair of tooth surfaces mesh continuously with a contact line and the basic relationships between tooth surfaces and their relative motion were derived mathematically. In addition, the relative normal curvature and the geodesic torsion of the tooth surfaces and relative motion were shown to be related to hypoid gears, and the EulerSavary law was extended to the equation of three-dimensional tooth surfaces for the most general form of gearing.
\end{abstract}

Key Words: Machine Element, Gear, Gear Mesh, Line Contact, Euler-Savary Equation, Normal Curvature, Geodesic Torsion

\section{Introduction}

When the tooth profile curvature of either member is chosen arbitrarily, the profile of the other one can be derived by applying the fundamental law called the "Euler-Savary equation"(1)". It is a very useful method, but it can only be utilized for spur and helical gear tooth action in a transverse plane.

In order to study three-dimensional gear mesh, this paper introduces the conditions that are necessary for gearing with a contact line that is along the pitch line: the first one is that the relative normal velocity is zero at any point of contact and the second one is that the normal vectors of the tooth surfaces at the contact point must fit together after small revolutions of the gear axes.

From the above conditions, two basic equations were obtained by using the normal curvatures of surface along the geodesic line and the geodesic torsion on their conjugate tooth surfaces. In addition, it

* Received, 16th February, 1998

** Faculty of Engineering, Toyama University 3190 Gofuku, Toyama-shi 930-8555, Japan. E-mail : noritoh@eng.toyama-u.ac.jp

*** Takahashi Hypoid Technical Research Institute 514-23 Shimosueyoshi, Tsurumi-ku, Yokohama-shi 230-0012, Japan was found that the Euler-Savary equation is a special example of a new three-dimensional basic relationship of hypoid gears.

\section{Relative Motion of Two Surfaces}

When two surfaces mesh and have a same contact line, we will describe here some of the basic relationships underlying these surfaces and conjugate action.

Figure 1 shows two surfaces which slide and roll on a contact line. They are denoted by vectors $\boldsymbol{X}_{1}$ and $\boldsymbol{X}_{2}$. When surface $\boldsymbol{X}_{\mathbf{2}}$ is stationary, relative motion is simulated by the planetary motion of the surface $\boldsymbol{X}_{1}$. If we assume that two of the surfaces are in contact with line $C$ at any time $t$, after a very small amount of time $d t$, the relative motion is advanced so that the

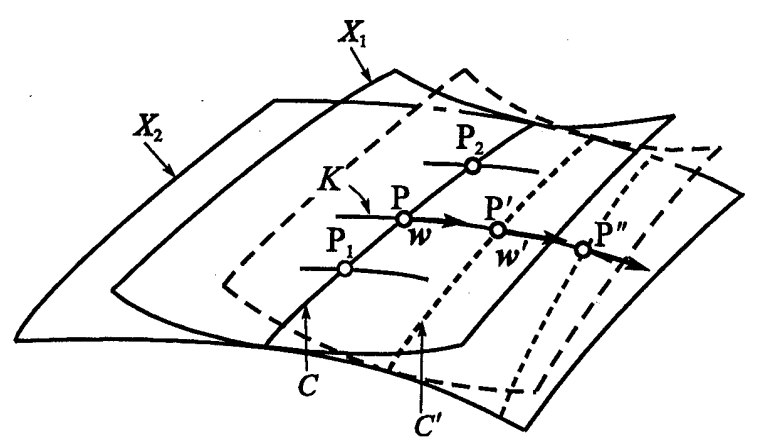

Fig. 1 Relative motion of two surfaces and a slide line 
contact line of these surfaces is $C^{\prime}$ on the surface $\boldsymbol{X}_{2}$. Then, relative velocity occurs on contact line $C$ due to the relative motion and similarly also occurs on the new contact line $C^{\prime}$. Vector $\boldsymbol{w}$ is the relative velocity at mean point $\mathrm{P}$ on line $C$. Now, we can consider line $K$, which comes into contact with vector $\boldsymbol{w}$ at point $\mathrm{P}$, on surface $\boldsymbol{X}_{2}$. Line $K$ intersects line $C^{\prime}$ at point $\mathrm{P}^{\prime}$. We can also make line $K$ comes into contact with relative velocity vector $\boldsymbol{w}^{\prime}$ at point $\mathrm{P}^{\prime}$. This type of line is a slide line. The same can be said in regard to the other points $P_{1}$ and $P_{2}$ which are along contact line $C$. Therefore, there are numerous slide lines on surface $\boldsymbol{X}_{2}$.

If two surfaces are replaced by two tooth surfaces, the standard point that is used to design and manufacture gears is chosen on the mean point of tooth surface. Here, let the mean point take the point being considered. In Fig. 1 the point being considered is point $\mathrm{P}$. Slide line $K$ which passes through point $\mathrm{P}$ is called a tooth spiral or a tooth trace. When surface $\boldsymbol{X}_{1}$ is stationary and the other surface $\boldsymbol{X}_{2}$ is moved, there is another slide line which passes through point $\mathrm{P}$ on tooth surface $\boldsymbol{X}_{1}$. The slide line is the tooth spiral of $\boldsymbol{X}_{1}$. As mentioned above, two tooth surfaces which have the same contact line and have relative motion each other produce a pair of tooth spirals. This pair of tooth spirals comes into contact at point $\mathrm{P}$, but the contact point subsequently proceeds with relative motion. Consequently, contact points describe a locus in space. The locus line is called a pitch line. In addition, two surfaces are described by a revolution of the pitch line around each gear axis. These surfaces are referred to as pitch surfaces ${ }^{(2)}$.

\section{Curve and Geodesic Line on Surface}

Figure 2 illustrates the tooth surface and the curve that is on it. The surface is denoted by vector $\boldsymbol{X}_{i}$. The point being considered is $\mathrm{P}$, and the curve which passes through point $\mathrm{P}$ is denoted by vector $\boldsymbol{X}_{i}\left(S_{i}\right)$. A geodesic line which comes into contact with

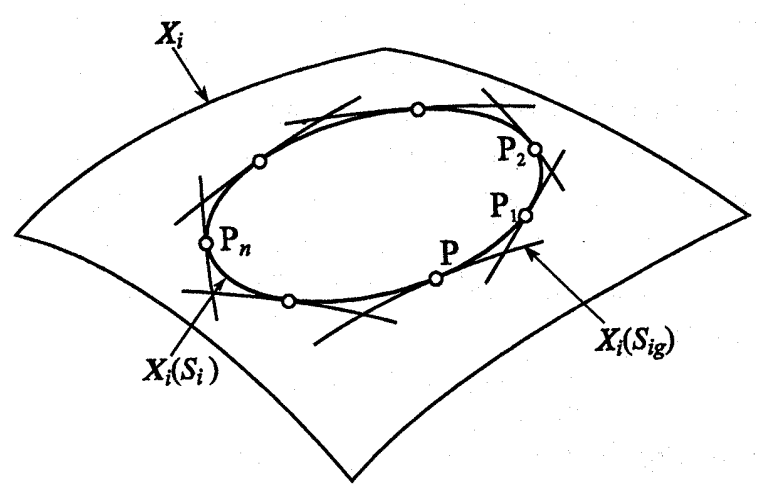

Fig. 2 Curve and geodesic line curve $\boldsymbol{X}_{i}\left(S_{i}\right)$ at point $\mathrm{P}$ is denoted by vector $\boldsymbol{X}_{i}\left(S_{i g}\right)$, $S_{i}$ and $S_{i g}$, indicate the arc lengths of these curves respectively. Points $\mathrm{P}, \mathrm{P}_{1}, \mathrm{P}_{2}, \cdots, \mathrm{P}_{n}, \cdots$, are on curve $\boldsymbol{X}_{i}\left(S_{i}\right)$. Certain geodesic lines which come into contact with the curve at those points are drawn on the surface $\boldsymbol{X}_{i}$. When curve $\boldsymbol{X}_{i}\left(S_{i}\right)$ is closed, a polygon is produced by these geodesic lines. If the number of divisions of the curve are increased infinitely, the polygon itself becomes a curve. Consequently, an infinitesimal part of the curve on the surface can be considered to be an infinitesimal part of a geodesic line that is in contact with the curve. Therefore, the curve on the surface should have its own the curvature and the torsion and also curvature and torsion of the geodesic line which comes into contact with the curve. The curvature and the torsion of the geodesic line are the normal curvature and geodesic torsion that are in the tangent direction of the curve at the point being considered. Therefore, if we consider the curve to be a tooth spiral, the characteristics of tooth surfaces are represented by geodesic lines $\boldsymbol{X}_{i}\left(S_{i g}\right)$ which come into contact with the tooth spirals.

\section{Normal and Geodesic Curvature of Tooth Surface}

We used the above characteristics of the tooth surfaces in order to obtain certain relationships between these surfaces and the relative motion. Then, the tooth surfaces mesh while touching a contact line.

Figure 3 shows two tooth surfaces which have meshed with a line contact. They are denoted by vectors $\boldsymbol{X}_{1}$ and $\boldsymbol{X}_{2}$. They are fixed on the gear axes, and they rotate together due to angular velocity vectors $\boldsymbol{\omega}_{1}$ and $\boldsymbol{\omega}_{2}$. We will consider a three right-

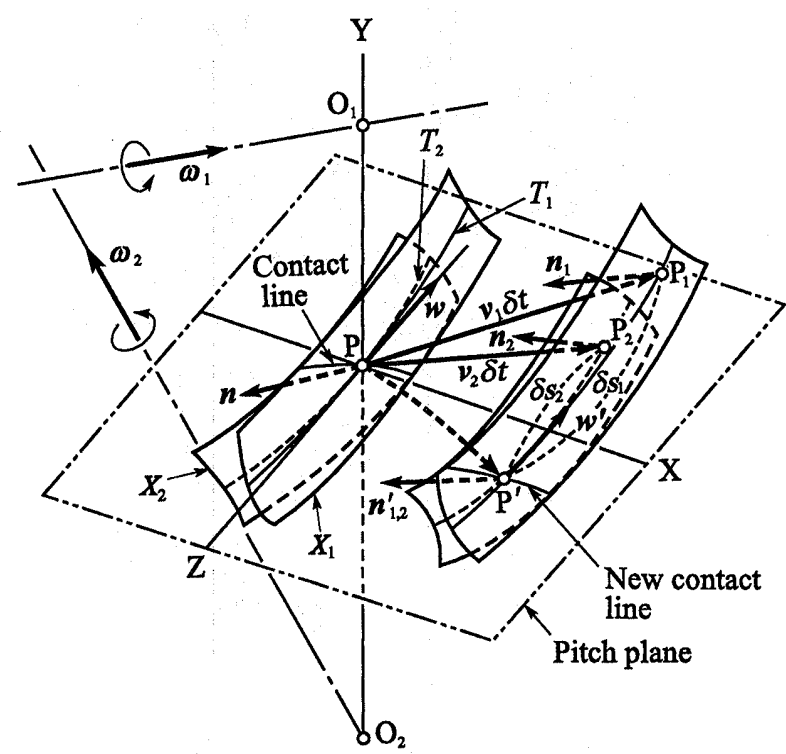

Fig. 3 Tooth surface mesh with a contact line 
hand orthogonal coordinate system $\mathrm{P}-\mathrm{XYZ}$ which is necessary in order to study the motion of tooth spirals on the tooth surface. Let $\mathrm{P}$ point being considered be origin $\mathrm{P}$ at any one instant $t . \mathrm{P}$ is the pitch point and the $Z$-axis is the relative velocity direction of a tooth spiral at point $\mathrm{P}$. The $\mathrm{Y}$-axis is the direction of vector product $\boldsymbol{v}_{2} \times \boldsymbol{v}_{1}$, where $\boldsymbol{v}_{1}$ and $\boldsymbol{v}_{2}$ are the velocity vectors for the pinion and the gear. $\boldsymbol{v}_{1}$ and $\boldsymbol{v}_{2}$ lie in the pitch plane. The $\mathrm{X}$-axis is perpendicular to the $\mathrm{Y}$ and $Z$ axes. The $Z X$ plane then becomes a pitch plane. The $\mathrm{Y}$-axis intersects with the two points $\mathrm{O}_{1}$ and $\mathrm{O}_{2}$ on the pinion and gear axes, respectively. Pitch point $\mathrm{P}$ at any time $t$ proceeds to a new pitch point $\mathrm{P}^{\prime}$ after a very small increment of two gear rolls. Then, point $\mathrm{P}$ on tooth surface $\boldsymbol{X}_{1}$ moves to point $\mathrm{P}_{1}$, and, similarly, the point $\mathrm{P}$ on tooth surface $\boldsymbol{X}_{2}$ moves to point $\mathrm{P}_{2}$. When the common normal vector of the tooth surfaces at point $\mathrm{P}$ is denoted by unit vector $\boldsymbol{n}, \boldsymbol{n}$ also moves to point $\mathrm{P}_{1}$ on surface $\boldsymbol{X}_{1}$ and to point $\mathrm{P}_{2}$ on surface $\boldsymbol{X}_{2}$ after a very small rotation increment and changes unit normal vectors $\boldsymbol{n}_{1}$ and $\boldsymbol{n}_{2}$ respectively. When the first contact point $\mathrm{P}$ on the tooth spirals proceeds to the next contact point $\mathrm{P}^{\prime}$, points $\mathrm{P}_{1}$ and $\mathrm{P}_{2}$ move along the respective tooth spirals $T_{1}$ and $T_{2}$ and they meet at the same point $\mathrm{P}^{\prime}$. At point $\mathrm{P}^{\prime}$, the normal vectors on their surfaces are denoted by $\boldsymbol{n}_{1}^{\prime}$ and $\boldsymbol{n}_{2}^{\prime}$, respectively. These two normal vectors must coincide. Therefore, the contact point moves by a small incremental arc length $d s_{1}$ along tooth spiral $T_{1}$ on tooth surface $\boldsymbol{X}_{1}$ and moves by a small incremental arc length $d s_{2}$ along tooth spiral $T_{2}$ on tooth surface $\boldsymbol{X}_{2}$. Arc $\overparen{\mathrm{PP}}^{\prime}$ is on the pitch line.

Figure 4 shows the motion on the pitch plane that

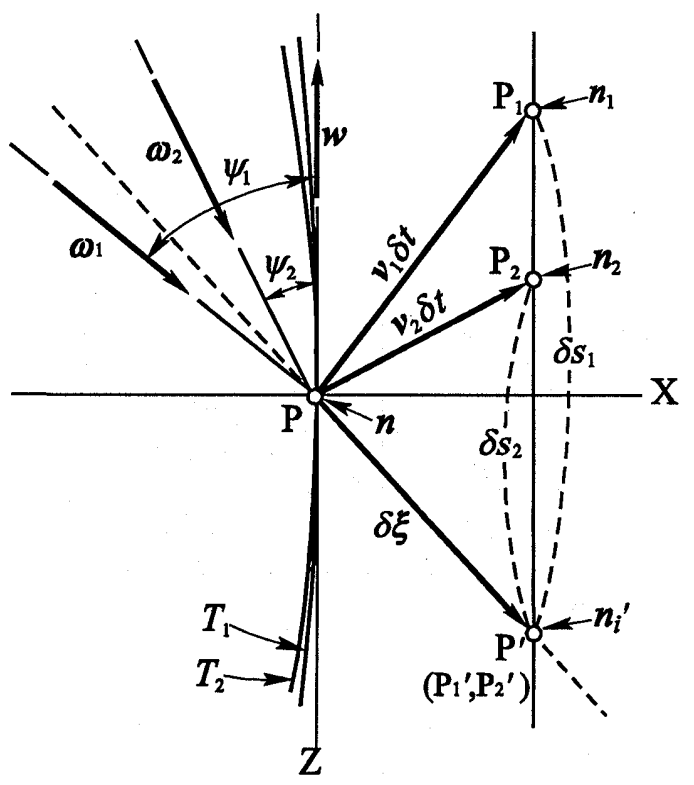

Fig. 4 Tooth spiral after small incremental relative motion is shown in Fig. 3. Angular velocity vectors $\boldsymbol{\omega}_{1}$ and $\boldsymbol{\omega}_{2}$ are projected onto the pitch plane. Relative velocity $\boldsymbol{w}$ at point $\mathrm{P}$ is vector $\boldsymbol{v}_{1}-\boldsymbol{v}_{2}$. Spiral angles $\psi_{1}$ and $\psi_{2}$ of the tooth spirals are the angles between the $Z$-axis and the respective $\omega_{1}$ and $\omega_{2}$ on the pitch plane. If vector $\overrightarrow{\mathrm{PP}^{\prime}}=d \xi, d \xi$ is the vector which indicates the direction of the pitch line.

The following equations were obtained based on the above data. Hereafter, we will use the subscript $i$. $i=1$ is the symbol for tooth surface $\boldsymbol{X}_{1}$ and $i=2$ is the symbol for tooth surface $\boldsymbol{X}_{2}$.

First, the normal vector $\boldsymbol{n}_{i}^{\prime}$ is given by the following equation :

$$
\boldsymbol{n}_{i}^{\prime}=\boldsymbol{n}+\boldsymbol{\omega}_{i} \times \boldsymbol{n} d t+\left[\frac{d \boldsymbol{n}_{i}}{d s_{i}}\right]_{\mathrm{P}} d s_{i}
$$

where []$_{\mathrm{P}}$ in the equation expresses the value of pitch point $\mathrm{P}$. Using $\triangle \mathrm{PP}_{i} \mathrm{P}^{\prime}$ in Fig. 4 , the following equation are obtained:

$$
d \boldsymbol{\xi}=\boldsymbol{v}_{i} d t-\frac{\boldsymbol{w}}{w} \dot{d}_{i}
$$

where $w=|\boldsymbol{w}|$.

Next, the relative velocity vector $\boldsymbol{w}^{\prime}$ of the tooth spirals at new pitch point $\mathrm{P}^{\prime}$ is given by the following equation:

$$
\boldsymbol{w}^{\prime}=\boldsymbol{w}+\boldsymbol{\omega} \times d \boldsymbol{\xi}
$$

where $\boldsymbol{\omega}$ is the relative angular velocity vector and $\boldsymbol{\omega}$ $=\omega_{1}-\omega_{2}$. At the first contact point $\mathrm{P}$ of the two tooth surfaces, the following well-known meshing condition must be satisfied.

$$
\boldsymbol{n} \cdot \boldsymbol{w}=0
$$

Moreover, at the new contact point $\mathrm{P}^{\prime}$ after a small incremental rotation, the following condition must be satisfied:

$$
\boldsymbol{n}^{\prime} \cdot \boldsymbol{w}^{\prime}=0
$$

Another necessary condition is the coincidence of the directions of the normal vectors $\boldsymbol{n}_{1}^{\prime}$ and $\boldsymbol{n}_{2}^{\prime}$ on the two tooth surfaces.

$$
\boldsymbol{n}_{1}^{\prime} \times \boldsymbol{n}_{2}^{\prime}=0
$$

From Eqs. (1) and ( 6 ), we can obtain

$$
\begin{gathered}
\left\{\boldsymbol{n} \times\left(\boldsymbol{\omega}_{2} \times \boldsymbol{n}\right)-\boldsymbol{n} \times\left(\boldsymbol{\omega}_{1} \times \boldsymbol{n}\right)\right\} \delta t+\boldsymbol{n} \\
\times \frac{d \boldsymbol{n}_{2}}{d s_{2}} \delta s_{2}-\boldsymbol{n} \times \frac{d \boldsymbol{n}_{1}}{d s_{1}} \delta s_{1}=0
\end{gathered}
$$

In this equation, $d \boldsymbol{n}_{i} / d s_{i}$ can be replaced by the $d \boldsymbol{n}_{i} / d s_{i g}$ from the geodesic line which is in contact with the tooth spiral at the point being considered, because $d s_{i}$ and $d s_{i g}$ are equal in the small incremental arc length. When normal curvature at point $\mathrm{P}$ is $1 / \rho_{i}$ and geodesic torsion is $1 / \tau_{i}$, we can obtain the next equation along the geodesic line using Frenet-Serret's formula as follows :

$$
\frac{d \boldsymbol{n}_{i}}{d s_{i}}=-\frac{1}{\rho_{i}} \frac{d \boldsymbol{X}_{i}}{d s_{i}}+\frac{1}{\tau_{i}} \frac{d \boldsymbol{X}_{i}}{d s_{i}} \times \boldsymbol{n}_{i}
$$

From Eqs. ( 7 ) and ( 8 ), we can obtain 


$$
\begin{gathered}
-\boldsymbol{n} \times(\boldsymbol{\omega} \times \boldsymbol{n}) \delta t-\boldsymbol{n} \times \frac{\boldsymbol{w}}{w}\left(\frac{1}{\rho_{1}} \delta s_{1}-\frac{1}{\rho_{2}} \delta s_{2}\right) \\
+\boldsymbol{n} \times\left(\frac{\boldsymbol{w}}{w} \times \boldsymbol{n}\right)\left(\frac{1}{\tau_{1}} \delta s_{1}-\frac{1}{\tau_{2}} \delta s_{2}\right)=0
\end{gathered}
$$

Thus, from Eq. ( 9 ), the relationship between the two normal curvatures from the tooth spiral direction on the tooth surface is obtained as follows:

$$
-\boldsymbol{\omega} \cdot \frac{\boldsymbol{w}}{w} \times \boldsymbol{n} \delta t+\frac{1}{\rho_{1}} \delta s_{1}-\frac{1}{\rho_{2}} \delta s_{2}=0
$$

Similarly, the relationship between the two geodesic curvatures is obtained as follows :

$$
-\boldsymbol{w} \cdot \boldsymbol{\omega} \delta t+w\left(\frac{1}{\tau_{1}} \delta s_{1}-\frac{1}{\tau_{2}} \delta s_{2}\right)=0
$$

Equations (10) and (11) are the results from Eq. (6).

Next, let us consider the significance of Eq. (5). Substituting Eq. ( 8 ) into Eq. (5) and arranging Eq. ( 5 ), the following equation can be obtained:

$$
\boldsymbol{n} \cdot\left(\boldsymbol{\omega}_{1} \times \boldsymbol{v}_{2}-\boldsymbol{\omega}_{2} \times \boldsymbol{v}_{1}\right) \delta t+\left(\frac{1}{\rho_{i}}-\frac{\boldsymbol{n} \cdot \boldsymbol{\omega} \times \boldsymbol{w}}{w^{2}}\right) w \delta s_{i}=0
$$

Here $p, q$, and $\lambda$ are given by the following scalar equations:

$$
\begin{aligned}
& p=\frac{\boldsymbol{n} \cdot \boldsymbol{\omega} \times \boldsymbol{w}}{w^{2}} \\
& q=\frac{\boldsymbol{n} \cdot\left(\boldsymbol{\omega}_{2} \times \boldsymbol{v}_{1}-\boldsymbol{\omega}_{1} \times \boldsymbol{v}_{2}\right)}{w^{2}} \\
& \lambda=\frac{\boldsymbol{\omega} \cdot \boldsymbol{w}}{w^{2}}
\end{aligned}
$$

Using Eqs. (13)-(15), Eq. (12) is written as follows :

$$
\delta t=\frac{1}{w} \frac{\frac{1}{\rho_{i}}-p}{q} \delta s_{i}
$$

Substituting Eqs. (10) and (11) which were obtained from Eq. (6) into Eq. (16) which was obtained from Eq. (5), the following important equations were obtained :

$$
\begin{aligned}
& \frac{1}{\frac{1}{\rho_{1}}-p}-\frac{1}{\frac{1}{\rho_{2}}-p}=\frac{1}{q} \\
& \frac{\frac{1}{\tau_{1}}}{\frac{1}{\rho_{1}}-p}-\frac{\frac{1}{\tau_{2}}}{\frac{1}{\rho_{2}}-p}=\frac{\lambda}{q}
\end{aligned}
$$

Equations (17) and (18) are the basic relationships between the normal curvatures and the geodesic torsions along the tooth spirals on the tooth surfaces. The contact point proceeds along the pitch line with the small incremental motion of tooth surfaces meshing, so these equations must be satisfied at all of the points on the pitch line. In Eq. (17) $q=0$ is the singular example. It is the turning point of interference of the tooth spirals that gives the concept ${ }^{(3)}$ of the limit normal curvature and the limit pressure angle. By rewriting Eq. (17), the following equation is obtained :

$$
\frac{1}{\rho_{1}-\frac{1}{p}}-\frac{1}{\rho_{2}-\frac{1}{p}}=-\frac{p^{2}}{q}
$$

This equation can be used in place of Eq. (17).

\section{Application to Concrete Gears}

\section{1 Hypoid gears}

Hypoid gears are hyperboloidal gears that have a conical external form and they are the most generalized gear pair. Therefore, the equations from the basic relationship obtained above can be applied to hypoid gears. Here, let us express $p, q$, and $\lambda$ using the hypoid gear elements.

$p, q$, and $\lambda$ are constants which are determined by a pair of pitch surface forms and normal direction of tooth surfaces. The pitch surfaces of the hypoid gears can be described by rotating the pitch line about each gear axis, as was mentioned above. The pitch surfaces can be replaced by their inscribed or circumscribed cones; these are referred to as pitch cones. The characteristics of these pitch cones are represented by the three elements of pitch cone distance, pitch angle, and the spiral angle of the tooth spiral. These are three cone elements.

Figure 5 shows the pitch cones of the gear and pinion and their three elements. $A_{1}$ and $A_{2}$ are the pitch cone distances, $\gamma$ and $\Gamma$ are the pinion and gear pitch angles, and $\psi_{1}$ and $\psi_{2}$ are the pinion and gear spiral angles. The coordinate system is the kinematics coordinate system $\mathrm{P}-\mathrm{XYZ}$. Each element contained equations of $p, q$, and $\lambda$, which are obtained as follows:

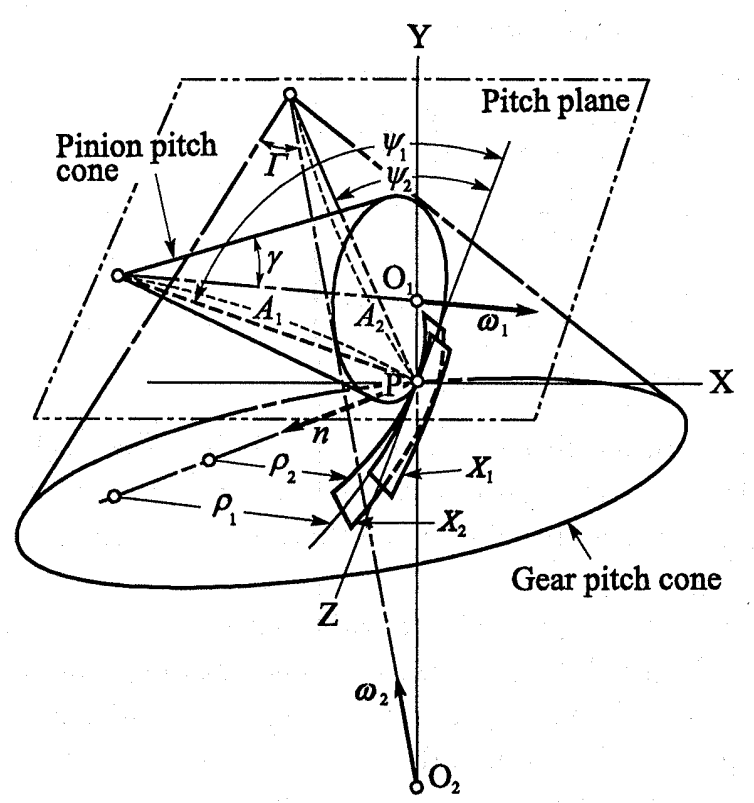

Fig. 5 Pitch cones of hypoid gears on their pitch plane 


$$
\begin{aligned}
& \omega_{1}=\omega_{1}\left[\begin{array}{c}
\cos \gamma \sin \phi_{1} \\
\sin \gamma \\
\cos \gamma \cos \psi_{1}
\end{array}\right] \\
& \boldsymbol{\omega}_{2}=\omega_{2}\left[\begin{array}{c}
\cos \Gamma \sin \psi_{2} \\
-\sin \Gamma \\
\cos \Gamma \cos \psi_{2}
\end{array}\right] \\
& \boldsymbol{v}_{1}=\omega_{1} A_{1} \sin \gamma\left[\begin{array}{c}
\cos \psi_{1} \\
0 \\
-\sin \psi_{1}
\end{array}\right] \\
& \left.\boldsymbol{v}_{2}=-\omega_{2} A_{2} \sin \Gamma\left[\begin{array}{c}
\cos \psi_{2} \\
0 \\
-\sin \psi_{2}
\end{array}\right]\right] \\
& \boldsymbol{\omega}=\boldsymbol{\omega}_{1}-\boldsymbol{\omega}_{2} \\
& =v_{n}\left[\begin{array}{c}
\tan \phi_{1} /\left(A_{1} \tan \gamma\right)+\tan \phi_{2} /\left(A_{2} \tan \Gamma\right) \\
1 /\left(A_{2} \cos \phi_{1}\right)-1 /\left(A_{2} \cos \phi_{2}\right) \\
1 /\left(A_{1} \tan \gamma\right)+1 /\left(A_{2} \tan \Gamma\right)
\end{array}\right] \\
& \boldsymbol{w}=\boldsymbol{v}_{1}-\boldsymbol{v}_{2} \\
& =v_{n}\left(\tan \psi_{1}-\tan \psi_{2}\right)\left[\begin{array}{c}
0 \\
0 \\
-1
\end{array}\right] \\
& v_{n}=\omega_{1} A_{1} \sin \gamma \cos \phi_{1}=-\omega_{2} A_{2} \sin \Gamma \cos \phi_{2}
\end{aligned}
$$

When $\phi$ is the pressure angle of the tooth surface at the pitch point, unit normal vector $n$ of the tooth surface is expressed by the following matrix :

$$
\boldsymbol{n}=\left[\begin{array}{c}
-\cos \phi \\
-\sin \phi \\
0
\end{array}\right]
$$

From the results above, $p, q$, and $\lambda$ are written as follows :

$$
\begin{aligned}
p & =\frac{-v_{1} \sin \phi+v_{2} \cos \phi}{\tan \phi_{1}-\tan \phi_{2}} \\
q & =\frac{v_{3} \cos \left(\phi_{1}-\phi_{2}\right) \sin \phi+v_{4} \cos \phi}{\cos \phi_{1} \cos \psi_{2}\left(\tan \phi_{1}-\tan \psi_{2}\right)^{2}} \\
\lambda & =\frac{-v_{3}}{\tan \phi_{1}-\tan \psi_{2}}
\end{aligned}
$$

where

$$
\begin{aligned}
& \nu_{1}=\frac{\tan \psi_{1}}{A_{1} \tan \gamma}+\frac{\tan \psi_{2}}{A_{2} \tan \Gamma} \\
& \nu_{2}=\frac{1}{A_{1} \cos \psi_{1}}-\frac{1}{A_{2} \cos \psi_{2}} \\
& \nu_{3}=\frac{1}{A_{1} \tan \gamma}+\frac{1}{A_{2} \tan \Gamma} \\
& \nu_{4}=\frac{\sin \psi_{1}}{A_{2}}-\frac{\sin \psi_{2}}{A_{1}}
\end{aligned}
$$

Substituting these $p, q$, and $\lambda$ values into Eqs. (17) and (18), we can quantitatively find the normal curvature and geodesic torsion of the tooth spiral of the hypoid gears.

\section{2 Gear pair with parallel axes}

When the hypoid axes become parallel, spur gears are considered to be a special type of hypoid gears. In regard to the method that makes the gear axes parallel, there are two cases as follows :

5.2.1 The pitch cylinder Here, the values of pitch angles $\gamma$ and $\Gamma$ of the hypoid gears are zero, in addition, the values of the spiral angles $\psi_{1}$ and $\phi_{2}$ are also equal. We will substitute $\gamma=0, \Gamma=0$, and $\phi_{1}=\phi_{2}$ $=\psi$ into Eqs. (20)-(28) with respect to the hypoid gears. Then, the radii of the pinion and the gear pitch cones at pitch point $\mathrm{P}$ are defined as the radii of the pitch circles. They are represented by $R_{1}$ and $R_{2}$, respectively. We have $R_{1}=A_{1} \sin \gamma$ and $R_{2}=A_{2} \sin \Gamma$. In cylindrical gears radii $R_{1}$ and $R_{2}$ of the pitch circles are constant and the pitch plane is a common tangent plane that is in contact with two pitch cylinders. Hence we can obtain the following equations:

$$
\left.\begin{array}{l}
\nu_{1}=\tan \phi\left(\frac{1}{R_{1}}+\frac{1}{R_{2}}\right) \\
\nu_{2}=0 \\
\nu_{3}=\frac{1}{R_{1}}+\frac{1}{R_{2}} \\
\nu_{4}=0 \\
\frac{p^{2}}{q}=\sin \phi \sin ^{2} \phi\left(\frac{1}{R_{1}}+\frac{1}{R_{2}}\right)
\end{array}\right\}
$$

Substituting Eq. (29) into Eq. (19), the following equation is obtained.

$$
\frac{1}{\rho_{1}}-\frac{1}{\rho_{2}}=-\sin \phi \sin ^{2} \phi\left(\frac{1}{R_{1}}+\frac{1}{R_{2}}\right)
$$

Similarly, from Eqs. (18) and (29), we can obtain

$$
\frac{1}{\tau_{1}}-\frac{1}{\tau_{2}}=-\sin \psi \cos \psi\left(\frac{1}{R_{1}}+\frac{1}{R_{2}}\right)
$$

Equation (30) expresses the relative curvature of the tooth spirals and Eq. (31) expresses their relative torsion. Equations (30) and (31) are the necessary conditions for gear pair meshing with a contact line.

Figure 6 shows the pitch cylinders and the pitch plane of the helical gears. Two tooth surfaces $\boldsymbol{X}_{1}$ and

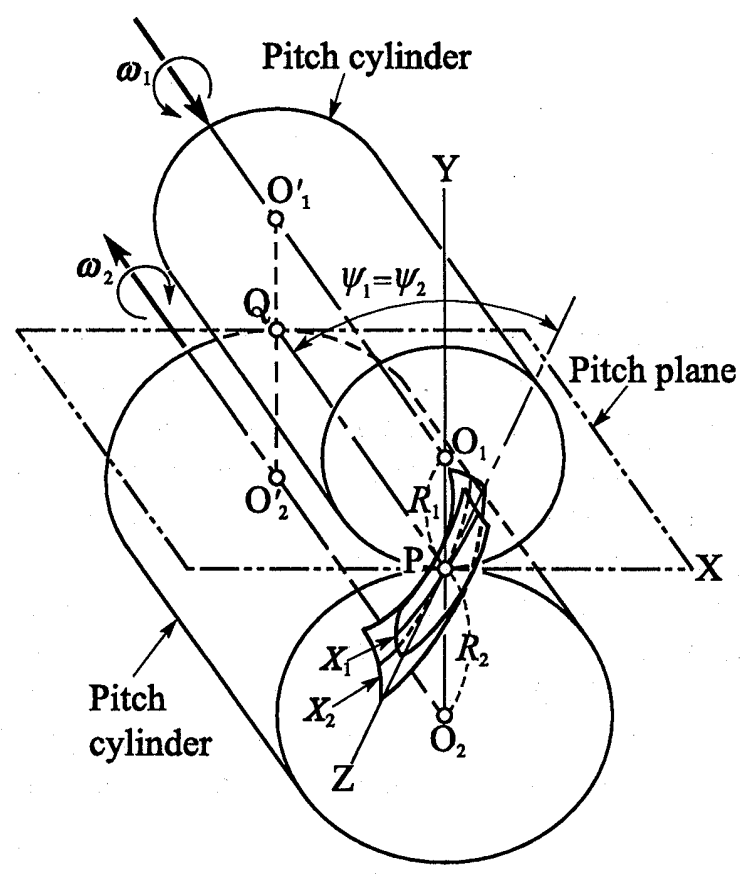

Fig. 6 Pitch cylinder 
$\boldsymbol{X}_{2}$ come into contact at pitch point $\mathrm{P}$. Points $\mathrm{Q}, \mathrm{O}_{1}^{\prime}$ and $\mathrm{O}_{2}^{\prime}$ correspond to points $\mathrm{P}, \mathrm{O}_{1}$ and $\mathrm{O}_{2}$, respectively. All other symbols are the same as defined previously.

5.2.2 Pitch plane Here, the values of pitch angles $\gamma$ and $\Gamma$ of the hypoid gears come together and become $\pi / 2$. Two gear axes are parallel and have the same directions. They mesh as if they are internal gears. If we calculate the equations for the hypoid gears under the above conditions, we can obtain the following equations :

$$
\begin{aligned}
\nu_{1} & =0 \\
\nu_{2} & =\frac{1}{A_{1} \cos \psi_{1}}-\frac{1}{A_{2} \cos \psi_{2}} \\
\nu_{3} & =0 \\
\nu_{4} & =\frac{\sin \psi_{1}}{A_{2}}-\frac{\sin \psi_{2}}{A_{1}} \\
p & =\left(\frac{1}{A_{1} \cos \psi_{1}}-\frac{1}{A_{2} \cos \psi_{2}}\right) \frac{\cos \phi}{\tan \psi_{1}-\tan \psi_{2}} \\
q & =\frac{\cos \phi}{\cos \psi_{1} \cos \psi_{2}\left(\tan \psi_{1}-\tan \psi_{2}\right)^{2}} \\
& \times\left(\frac{\sin \psi_{1}}{A_{2}}-\frac{\sin \psi_{2}}{A_{1}}\right) \\
\lambda & =0
\end{aligned}
$$

Whence, $1 / p$ is given by the following equation.

$$
\frac{1}{p}=\frac{A_{1} A_{2} \sin \left(\psi_{1}-\psi_{2}\right)}{\left(A_{2} \cos \phi_{2}-A_{2} \cos \psi_{2}\right) \cos \phi}
$$

Figure 7 shows pinion and gear tooth surfaces $\boldsymbol{X}_{1}$ and $\boldsymbol{X}_{2}$, whose pitch surfaces are a plane and which come into contact at pitch point $\mathrm{P}$. The pinion and gear axes intersect the pitch plane at points $\mathrm{O}_{\mathrm{P}}$ and $\mathrm{O}_{\mathrm{G}}$. Therefore we can get $\overline{\mathrm{O}_{\mathrm{P}} \mathrm{P}}=A_{1}$ and $\overline{\mathrm{O}_{\mathrm{G}} \mathrm{P}}=A_{2}$. Let the intersection of the extension of line $\overline{\mathrm{O}_{\mathrm{P}} \mathrm{O}_{\mathrm{G}}}$ and the $\mathrm{X}-$ axis be point $\mathrm{O}$ and let $\overline{\mathrm{OP}}=r, \overline{\mathrm{OO}_{\mathrm{P}}}=R_{\mathrm{I}}$, and $\overline{\mathrm{OO}_{\mathrm{G}}}=$ $R_{\mathrm{II}}$. Now, when straight line $\overline{\mathrm{O} u}$ is drawn at right angles to $\overline{\mathrm{OO}_{\mathrm{P}}}$ on the pitch plane, let $\angle \mathrm{PO} u=\alpha, \tan \alpha$ is given by the following equation:

$$
\tan \alpha=\frac{r-A_{2} \sin \phi_{2}}{A_{2} \cos \psi_{2}}
$$

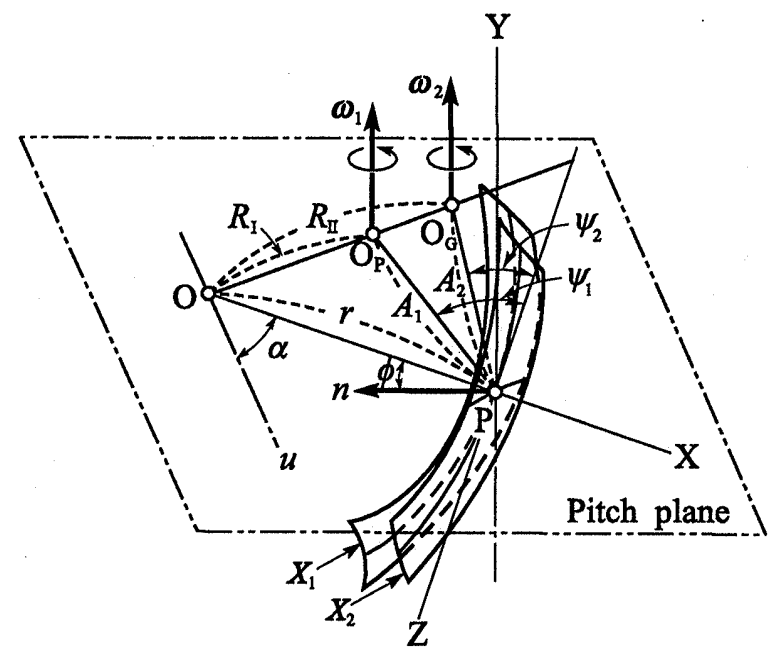

Fig. 7 Pitch plane

$$
=\frac{A_{1} \sin \psi_{1}-A_{2} \sin \psi_{2}}{A_{2} \cos \psi_{2}-A_{1} \cos \psi_{1}}
$$

Whence, solving for $r$, we have

$$
r=\frac{A_{1} A_{2} \sin \left(\psi_{1}-\psi_{2}\right)}{A_{2} \cos \psi_{2}-A_{1} \cos \psi_{1}}
$$

From Eqs. (33) and (35), we can obtain

$$
\begin{aligned}
& \frac{1}{p}=\frac{r}{\cos \phi} \\
& \frac{p^{2}}{q}=\cot \alpha \cos \phi\left(\frac{1}{A_{1} \cos \psi_{1}}-\frac{1}{A_{2} \cos \psi_{2}}\right)
\end{aligned}
$$

Referring to Fig. 7, we have

$$
\left.\begin{array}{l}
R_{\mathrm{I}}=\frac{A_{1} \cos \phi_{1}}{\cos \alpha} \\
R_{\mathrm{II}}=\frac{A_{2} \cos \psi_{2}}{\cos \alpha}
\end{array}\right\}
$$

$R_{\mathrm{I}}$ and $R_{\mathrm{II}}$ are the radii of the pitch circles of a gear pair with parallel axes, and point $O$ corresponds to the new pitch point. From Eqs. (37) and (38), we have

$$
\frac{p^{2}}{q}=\frac{\cos \phi}{\sin \alpha}\left(\frac{1}{R_{\mathrm{I}}}-\frac{1}{R_{\mathrm{II}}}\right)
$$

Substituting Eqs. (36) and (39) into Eq.(19), we can obtain the following :

$$
-\frac{1}{\rho_{1} \cos \phi-r}+\frac{1}{\rho_{2} \cos \phi-r}=\frac{1}{\sin \alpha}\left(\frac{1}{R_{\mathrm{I}}}-\frac{1}{R_{\mathrm{II}}}\right)
$$

In addition, by substituting Eq.(18) into $\lambda=0$, we can obtain the following :

$$
\frac{\frac{1}{\tau_{1}}}{\frac{1}{\rho_{1} \cos \phi}-\frac{1}{r}}=\frac{\frac{1}{\tau_{2}}}{\frac{1}{\rho_{2} \cos \phi}-\frac{1}{r}}
$$

In Eqs. (40) and (41), $1 /\left(\rho_{1} \cos \phi\right)$ and $1 /\left(\rho_{2} \cos \phi\right)$ are the geodesic curvatures of the tooth spirals on the pitch plane and, in addition, they are equal to the curvatures of the plane curves. Therefore Eq. (40) concurs the Euler-Savary equation on the plane curve. Here $\phi$ corresponds to the spiral angle of the internal gear, and $\phi=0$ when we consider spur gears.

\section{Conclusion}

When the tooth surfaces mesh continuously after the infinitesimal turning motion with an instantaneous contact line, it is made clear by this study that there are two necessary conditions and two kinds relationships that are necessary for gearing. One of the necessary conditions is that the normal vectors at any contact point on their tooth surfaces should coincide and the other is that the relative normal velocity at the contact point must be zero.

Equations (17) and (18) can be derived algebraically by using these conditions. The former equations express the relationship between the normal curvatures and relative motion of the tooth surfaces and the latter equation is composed of geodesic torsions and the relative motion of their surfaces.

When the gear axes are parallel and have the 
same directions, a hypoid pair becomes spur gears. Here each pitch angle of the hypoid pair is just a right angle and each spiral angle becomes zero. At the same time, the tooth spirals of the hypoid gears change into the gear profiles on the pitch plane of the spur gears. Thus, Eq. (40) concurs the Euler-Savary equation.

In conclusion, there are two basic relationships required for three-dimensional gearing, and they can be applied to all other types of two-dimensional gearing.

\section{References}

(1) Dudly, D.W., Gear Handbook, (1962), p.1-7, McGraw-Hill.

(2) Takahashi, K. and Ito, N., Studies on Pitch Surface of Hypoid Gear, Trans. Jpn. Soc. Mech. Eng., (in Japanese), Vol. 49, No. 443, C(1983), p. 12461255.

(3) Wildhander, E., Basic Relationship of Hypoid Gears II, Am. Mach., Vol. 90, No. 2 (1946), p. 131134. 